\title{
Análisis de la amenaza por movimientos en masa detonados por sismo en los Andes colombianos, caso de estudio: Barbosa (Antioquia)*
}

\author{
Edier Vicente Aristizábal Giraldo - Federico José Gómez Cardonab $^{\mathrm{b}}$ \\ - Edwin Fabián García Aristizábalc - Juan Carlos Guzmán Martínez ${ }^{d}$
}

\begin{abstract}
Resumen: los sismos constituyen uno de los mayores detonantes de movimientos en masa en el mundo. Diversas metodologías han sido desarrolladas para predecir y evaluar estos fenómenos desde enfoques estadísticos, físicos o combinación de ambos. En el presente estudio se analiza la amenaza por movimientos en masa detonados por sismos en el municipio de Barbosa, ubicado en los Andes colombianos, a través de los dos métodos más implementados: el análisis seudoestático y el método de Newmark, debido a su fácil uso, larga trayectoria y aceptación investigativa en los análisis de equilibrio límite. Los resultados permiten entender la respuesta geomecánica del subsuelo ante sismos en condiciones tanto saturadas como secas. El caso de estudio presentado señala un gran potencial de las metodologías mencionadas como herramientas de análisis de la amenaza de movimientos en masa detonados por sismos en zonas montañosas. Como se ha presentado en diferentes estudios, el porcentaje de áreas inestables se encuentra directamente relacionado con el grado de saturación del terreno y la pendiente. Para el municipio de Barbosa, y utilizando el método de Newmark, el área inestable alcanza el 59\% del municipio en condiciones saturadas y $17 \%$ en condiciones secas; mientras que utilizando el método seudoestático representan el $22 \%$ y $8 \%$ del municipio, respectivamente.
\end{abstract}

* Artículo de investigación.

a Doctorado, Universidad Nacional de Colombia. Profesor asistente, Departamento de Geociencias y Medioambiente, Facultad de Minas. Universidad Nacional de Colombia, sede Medellín. Medellín, Colombia. Correo electrónico: evaristizabalg@unal.edu.co ORCID: https://orcid.org/0000-0002-2648-2197

b Ingeniero geólogo. Universidad Nacional de Colombia, sede Medellín. Medellín, Colombia. Correo electrónico: fejgomezca@unal.edu.co ORCID: http://orcid.org/0000-0002-8525-4354

c Doctorado, Universidad de Kyoto, Japón. Profesor Asociado, Facultad de Ingeniería, Universidad de Antioquia. Medellín, Colombia. Correo electrónico: edwin.garcia@udea.edu.co ORCID: https://orcid.org/0000-0002-4837-8518

d Ingeniero civil, Universidad de Antioquia. Medellín, Colombia. Correo electrónico: juanc.guzman@udea.edu.co oRciD: https://orcid.org/0000-0002-7066-2881 
Palabras clave: movimientos en masa; sismos; andes colombianos; método de Newmark; análisis seudoestático

Recibido: 10/03/2020 Aceptado: 28/05/2020

Disponible en línea: 09/12/2020

Cómo citar: E. V. Aristizábal Giraldo, F. J. Gómez Cardona, E. F. García Aristizábal, y J. C. Guzmán Martínez, «Análisis de la amenaza por movimientos en masa detonados por sismo en los Andes colombianos, caso de estudio: Barbosa (Antioquia)», Cien.Ing.Neogranadina, vol. 30, n. ㄹ jul. 2020.

\title{
Analysis of the Threat from Mass Wasting Triggered by Earthquakes in the Colombian Andes, Case Study: Barbosa (Antioquia)
}

\begin{abstract}
Earthquakes are one of the biggest triggers of mass wasting in the world. Various methodologies have been developed to predict and evaluate these phenomena from statistical or physical approaches or a combination of both. This study analyzes the threat of mass wasting triggered by earthquakes in the municipality of Barbosa, located in the Colombian Andes, through the two most widely implemented methods: pseudo-static analysis and Newmark's method, due to their easy use, long history and research acceptance in limit equilibrium analysis. The results allow us to understand the geomechanical response of the subsoil to earthquakes in both saturated and dry conditions. The case study presented indicates the great potential of the abovementioned methodologies as tools for analyzing the threat of mass wasting triggered by earthquakes in mountainous areas. As it has been presented in different studies, the percentage of unstable areas is directly related to the degree of the land saturation and the slope. For the municipality of Barbosa, and using Newmark's method, the unstable area reaches 59\% of the municipality in saturated conditions and $17 \%$ in dry conditions; while using the pseudostatic method they represent $22 \%$ and $8 \%$ of the municipality, respectively.
\end{abstract}

Keywords: mass wasting; earthquakes; Colombian Andes; Newmark's method; pseudostatic analysis 


\section{Introducción}

Los movimientos en masa son una de las amenazas con mayor capacidad destructiva [1]. Dichos movimientos en masa han causado decenas de miles de muertes y billones de dólares en pérdidas económicas en el siglo pasado [3], [4]. En Colombia, de acuerdo con Sánchez y Aristizábal [5], solo el 0,7\% de los movimientos en masa registrados entre los años 1900 y 2016 han sido detonados por sismos; sin embargo, han generado el 5\% de las víctimas fatales registradas por movimientos en masa. El evento sísmico con el mayor número de movimientos en masa registrado en Colombia se presentó al suroccidente de los Andes colombianos, el 6 de junio de 1994, donde un sismo de magnitud local $\mathrm{ML}=6,4$ y profundidad de $10 \mathrm{~km}$ generó un número estimado de 3.002 deslizamientos traslacionales, que formaron un flujo de lodos en la cuenca del río Páez, y un saldo aproximado de 1.100 muertos [6]. Específicamente en el Valle de Aburrá se han registrado sismos con intensidades intermedias y daños importantes en el año 1979 y 1992 [7], pero sin registros en las bases de datos DesInventar y Sistema de Información de Movimientos en Masa (simma). Sin embargo, el origen tectónico del valle [8], [9] e indicios geomorfológicos, especialmente asociados a la presencia de grandes depósitos de vertiente sobre la margen noroccidental del Valle de Aburrá, permiten proponer como factor detonante de dichos antiguos movimientos en masa la ocurrencia de sismos [9], [11].

Los sismos no son una amenaza ajena a nuestro territorio. Los Andes colombianos se encuentran en el Bloque Andino del Norte [12], [13], cuya evolución y expresión tectónica, geológica y geomorfológica es el resultado de interacciones complejas entre la placa de Sudamérica, las placas oceánicas Farallón, Caribe y Nazca-Cocos y bloques litosféricos como el bloque Panamá-Chocó y el bloque Norandino [13]-[16]. El movimiento relativo de estas placas ha generado la acreción de al menos cinco bloques litotectónicos que causan procesos de fallamiento, levantamiento, exhumación y depositación; procesos determinantes en la geomorfología característica del actual relieve [17]. Además de generar los terrenos montañosos de los Andes
Colombianos altamente susceptibles a la ocurrencia de movimientos en masa, genera una serie de sismofuentes interplaca como el denominado Nido de Bucaramanga, además de zonas de cizalla de escala kilométrica que abarcan varias unidades litotectónicas asociadas a sistemas de falla regionales, como el Sistema Cauca Romeral y Espíritu Santo [13], [18]. En este contexto se enmarca el valle de Aburrá, un valle de origen tectónico y escarpadas vertientes que ha estado activo tectónicamente por millones de años y tiene asociados importantes sistemas de falla como los anteriormente mencionados [19].

La zonificación de la amenaza por movimientos en masa implementa diversas metodologías de carácter heurístico [20]-[22], estadístico [23]-[25] o con base física [26]-[29]. Sin embargo, un número importante de dichas metodologías evalúa realmente la susceptibilidad, ya que no consideran el factor detonante. En cuanto a los métodos que incorporan en su análisis el factor detonante, estos han sido enfocados a la lluvia. Para el detonante sísmico, los métodos disponibles son escasos y han abordado clásicamente análisis seudoestáticos, análisis esfuerzo-deformación y análisis de desplazamientos acumulados permanentes [30]. El análisis seudoestático de laderas fue desarrollado inicialmente por Terzaghi [31], para modelar la sacudida sísmica como una fuerza permanente que es aplicada al diagrama de fuerzas del análisis convencional de equilibrio límite estático [30], y se ha convertido en uno de los análisis más usados debido a su simplicidad [32]. Los métodos esfuerzo-deformación permiten aproximaciones coherentes a las deformaciones permanentes que sufren las laderas ante un sismo; sin embargo, se requiere una exigente parametrización, con una alta sensibilidad a dichos valores de entrada. En cuanto a los métodos de desplazamiento permanentes, Newmark (1965) [33] propuso un método simple inicialmente concebido para embalses y represas, que permite modelar la ladera como un bloque rígido, que se desliza sobre un plano inclinado bajo una aceleración crítica conocida del sismo, arrojando el umbral requerido para superar la resistencia al corte y causar la falla [1], [30]. Existen también en el estado del arte algunas aproximaciones 
estadísticas [34]-[38], determinísticas [26], [33], [39] o combinación de ambas [40], [41] e incluso como parte de análisis multi-amenaza de estructuras [42].

En Colombia, se han elaborado recientes estudios en el Valle de Aburrá, específicamente en Medellín, considerando los sismos como factor detonante de movimientos en masa [43], [44]. En el presente trabajo se evalúa la amenaza por movimientos en masa detonados por sismos en el municipio de Barbosa, localizado en el Valle de Aburrá, utilizando el análisis seudoestático y el método de desplazamiento permanente. Los métodos se implementaron para diferentes áreas del municipio de acuerdo con la información disponible. Para los suelos rurales del municipio, donde se contaba con modelos de elevación de menor resolución espacial $(5 \mathrm{~m} \times 5 \mathrm{~m})$ e información de los parámetros del suelo a nivel regional (escala 1:10.000), se implementó el método de Newmark, que evalúa los desplazamientos acumulados permanentes. Para las áreas urbanas, donde se contaba con modelos de elevación a mayor detalle ( $2 \mathrm{~m} \times 2 \mathrm{~m})$ e información de los parámetros del suelo a partir de estudios puntuales, se implementó el análisis seudoestático. Los resultados permiten obtener una aproximación de las condiciones de amenaza y áreas de mayor exposición a movimientos en masa detonados por sismos para el municipio de Barbosa, de acuerdo con la escala y nivel de información disponible. Adicionalmente, la metodología presentada puede ser aplicada como herramienta de evaluación de la amenaza en otras áreas de los Andes colombianos, como insumo para la elaboración de los estudios básicos de amenaza exigidos para los Planes de Ordenamiento Territorial a escala rural $(1: 25.000)$ y urbana (1:5.000).

\section{Área de estudio}

El municipio de Barbosa se localiza en el denominado Valle de Aburrá, al noroccidente de los Andes Colombianos (Figura 1). Este territorio se encuentra en una región sísmica activa caracterizada como amenaza media por la Norma Sismorresistente (NSR-10) [45]. Estas condiciones de amenaza se suman a los altos niveles de exposición por la acelerada y poco planificada ocupación de las laderas [46], donde adicionalmente se presentan efectos de sitio y amplificación, debido a las características geotécnicas y geométricas de los suelos, conformados por espesos perfiles de meteorización y extensos depósitos de vertiente [45]. Por consiguiente, la evaluación de los efectos sísmicos en la estabilidad de las laderas ubicadas en el municipio de Barbosa es fundamental para la ordenación de su territorio debido a las condiciones susceptibles a este tipo de evento.

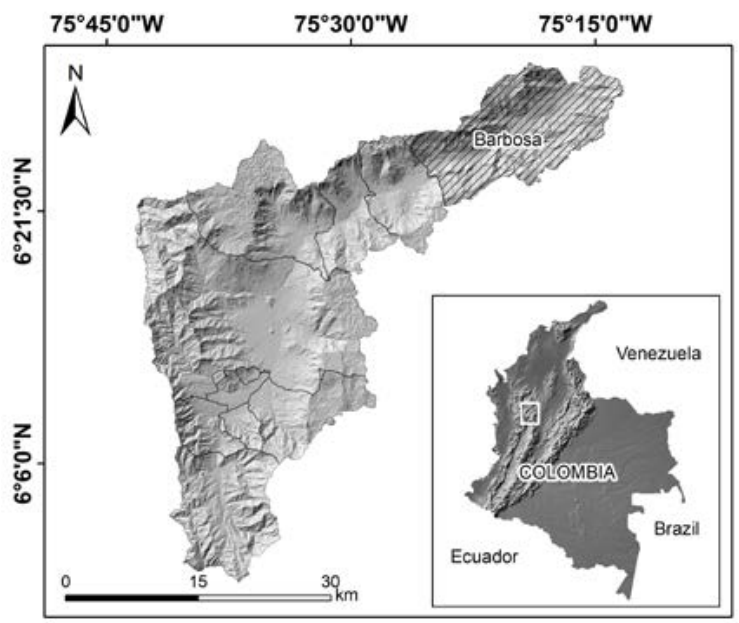

Figura 1. Localización del municipio de Barbosa.

Fuente: elaboración propia.

Barbosa está formado por un relieve montañoso con alturas entre 1.300 m.s.n.m. y 2.630 m.s.n.m., caracterizado por filos ramificados y alargados localmente deflectados, con pendientes de moderadas a fuertes y valles amplios y muy incisados. A medida que desciende hacia el fondo del valle, el relieve se suaviza, con filos de topes redondeados y planos, y vertientes moderadamente incisadas. La morfología del fondo del valle se caracteriza por estar asociada con los depósitos aluviales del río Aburrá y los conos de deyección de depósitos; sobre uno de estos conos está asentado el casco urbano del municipio [47]. Su clima corresponde al de un bosque húmedo subtropical con temperaturas promedio de $25^{\circ} \mathrm{C}$ y precipitación media anual de $2.400 \mathrm{~mm} /$ año. La geología se compone de unidades metamórficas, como esquistos y neises de 
los Esquistos de Cajamarca; ígneas, como granitos y tonalitas del Batolito antioqueño; depósitos de vertiente, subclasificados en depósitos de flujos de lodos y/o escombros y depósitos de movimientos en masa; depósitos aluviales, que principalmente corresponden a los depósitos del río Aburrá, y aluviotorrenciales de sus drenajes tributarios, además de llenos antrópicos [7], [18], [47].

\section{Metodología y datos}

\section{Análisis seudoestático}

El método seudoestático es una generalización del análisis de estabilidad por equilibrio límite, en el cual los efectos de los sismos son representados por una fuerza estática equivalente cuya magnitud es producto del coeficiente sísmico $\mathrm{k}$ y el peso de la masa que potencialmente se va a deslizar [48]. Este método evalúa los efectos sísmicos como una fuerza permanente unidireccional, mediante la simulación del incremento de las fuerzas inerciales debido a la aceleración de un sismo, suponiendo que las fuerzas sísmicas son proporcionales al peso de la masa deslizante. Usualmente, solo se adiciona la fuerza sísmica en la componente horizontal en el análisis de equilibrio límite y se consideran los efectos de las fuerzas verticales cercanos a cero [31]. Para calcular el Factor de Seguridad (Fs), Matasovic [49] propone la ecuación (1):

$F S=\frac{\frac{c}{\gamma_{s} z \cos ^{2} \theta}+\left[1-\frac{\left.\gamma_{w} z-h_{w}\right)}{\gamma_{s} z}\right]-k_{h} \tan \theta \tan \varphi}{k_{h}+\tan \theta}$

donde $C$ es la cohesión, $\varphi$ es el ángulo de fricción efectiva, $\gamma_{s}$ es el peso unitario de los materiales, $\theta$ es el ángulo de la pendiente, $z$ es el espesor del estrato deslizable, $\gamma_{\mathrm{w}}$ es el peso unitario del agua, $k_{h}$ es el coeficiente horizontal seudoestático y $h_{w}$ es la distancia al nivel freático medido desde la superficie.

Para la parametrización de las propiedades geomecánicas de los suelos que conforman el área urbana del municipio de Barbosa se utilizó la zonificación geotécnica elaborada en los estudios de microzonificación sísmica para el Valle de Aburrá, donde se realizaron perforaciones y ensayos de laboratorio con correlaciones de ensayos de penetración estándar [7], [18]. Esta zonificación se detalló a partir de información geotécnica suministrada por el municipio de estudios locales de suelos para propósitos constructivos. Para la pendiente se utilizó el modelo digital de elevación con resolución espacial de $2 \mathrm{~m}$ elaborado por el Instituto Geográfico Agustín Codazzi (IGAC) en el proyecto CartoAntioquia.

Para la selección del coeficiente horizontal seudoestático, se desarrolló la metodología propuesta por la Norma NSR-10 [50] para análisis seudoestáticos, en la cual se evalúa las condiciones sísmicas locales mediante las características de la zona de estudio. En el título $\mathrm{H}$ de la 10 se describe el diseño y estabilidad de taludes teniendo en cuenta los efectos sísmicos mediante el análisis seudoestático. Esta metodología plantea determinar el coeficiente sísmico horizontal seudoestático $\left(k_{h}\right)$ en función de la aceleración máxima del terreno $\left(A_{\max }\right)$, obtenida del espectro de diseño para el periodo de vibración cero establecida por el estudio de microzonificación sísmica del sitio. En el presente estudio se emplea el espectro de aceleración superficial para el periodo de retorno de 475 años y amortiguamiento del 5\%, tomado de los estudios de microzonificación sísmica detallada del municipio de Barbosa elaborados por [7].

La NSR-10 define las aceleraciones máximas de acuerdo con el espectro de diseño, donde presenta la formulación de la aceleración máxima del terreno para un periodo de vibración igual a cero, dada por la ecuación (2):

$$
K_{S T}=k_{h}=0.8 A_{\max }
$$

donde $\mathrm{A}_{\mathrm{a}}$ es la aceleración pico efectiva de diseño, $F_{a}$ es el coeficiente de amplificación que afecta la aceleración en la zona de períodos cortos e I representa el coeficiente de importancia. Estas variables fueron obtenidas a partir de los espectros de respuesta a nivel de superficie del terreno realizados por [7]. El coeficiente de importancia se determina a partir del uso de las edificaciones expuestas; en el presente proyecto se emplea el valor 
unitario, correspondiente al grupo de importancia I: estructuras de ocupación normal (Tabla 1).

Tabla 1. Valores del coeficiente de importancia

\begin{tabular}{ll} 
Grupo de Uso & Coeficiente de importancia, I \\
IV & $\frac{1,55}{1,25}$ \\
\hline III & $\frac{1,10}{1,00}$ \\
\hline II
\end{tabular}

Fuente: [7] y NSR-10.

A partir de $A_{\max }$ se obtiene el $k_{h}$ con la ecuación (3) teniendo en cuenta los criterios para el análisis seudoestático dados en la tabla de la NSR-10 [50, H.5.2.5]. Para este estudio se adopta el valor 0,8 que representa "Suelos, enrocados y macizos rocosos muy fracturados $\left(\mathrm{RQD}^{1}<50 \%\right)$ " (Tabla 2$)$.

$$
K_{S T}=k_{h}=0.8 A_{\max }
$$

Tabla 2. Valores de $\mathrm{K}_{\mathrm{ST}} / \mathrm{A}_{\max }$ mínimos para análisis seudoestático de taludes

\begin{tabular}{|c|c|c|}
\hline Material & $\frac{\mathrm{K}_{\mathrm{ST}}}{A_{\max }}$ & $\begin{array}{l}\text { Análisis de amplificación } \\
\text { mínimo }\end{array}$ \\
\hline $\begin{array}{l}\text { Suelos, enrocados y } \\
\text { macizos rocosos muy } \\
\text { fracturados (RQD }<50 \% \text { ) }\end{array}$ & 0,80 & Ninguno \\
\hline $\begin{array}{l}\text { Macizos rocosos } \\
(\mathrm{RQD}>50 \%)\end{array}$ & 1,00 & Ninguno \\
\hline $\begin{array}{l}\text { Todos los materiales } \\
\text { térreos }\end{array}$ & 0,67 & $\begin{array}{l}\text { Amplificación de onda } \\
\text { unidimensional en dos } \\
\text { columnas y promediar }\end{array}$ \\
\hline $\begin{array}{l}\text { Todos los materiales } \\
\text { térreos }\end{array}$ & 0,50 & $\begin{array}{l}\text { Amplificación de onda } \\
\text { bidimensional }\end{array}$ \\
\hline
\end{tabular}

Fuente: NSR-10.

Finalmente, los escenarios modelados con el análisis seudoestático corresponden a las condiciones seca y saturada del terreno, como escenarios que contrastan las condiciones de amenaza. Aunque ambos escenarios no corresponden al escenario más frecuente y probable, sí permiten

1 Por sus siglas en inglés, Rock Quality Designation. enmarcar las condiciones de amenaza del municipio entre el escenario de menor criticidad y el de mayor criticidad.

\section{Desplazamientos permanentes acumulados}

Este método ha dado útiles resultados modelando el comportamiento dinámico de las laderas a nivel regional [51], aunque no necesariamente predice los desplazamientos medidos, sino que establece un índice útil de cómo una ladera se podría comportar durante un evento sísmico [1].

En el presente trabajo se utilizó el método de Newmark [33]. Múltiples variaciones del método de Newmark han sido propuestas para obtener resultados precisos en términos de desplazamientos al modelar rigurosamente la respuesta dinámica de la ladera [1], [30], [52]. El método requiere evaluar el factor de seguridad, la aceleración crítica del terreno y la aceleración máxima en roca (Peak Ground Acceleration o PGA). Jibson ET AL. [1] proponen un modelo de equilibrio límite de talud infinito en material friccionante y cohesivo para obtener el factor de seguridad a partir de la ecuación (4):

$$
F S=\frac{c}{\gamma_{s} z \sin \theta}+\frac{\tan \varphi}{\tan \theta}-\frac{m \gamma_{w} \tan \varphi}{\gamma_{s} \tan \theta}
$$

donde $z$ es el espesor del suelo y $\mathrm{m}$ es la proporción del suelo que está saturado. Newmark [33] señala que la aceleración crítica $a_{c}$ de un bloque que potencialmente se puede deslizar es función del factor de seguridad estático y de la geometría del bloque expresado en la ecuación (5):

$$
a_{c}=(F S-1) \sin \theta
$$

donde $a_{c}$ es la aceleración crítica en términos de la aceleración de la gravedad g, y el ángulo que forma la horizontal con el centro de masa del bloque, que generalmente se aproxima a la pendiente.

Finalmente, a partir de $a_{c}$ yla aceleración máxima en roca (PGA) se calculan los desplazamientos de Newmark a través de una regresión logarítmica estimada por Jibson [52](2 en la ecuación (6):

$$
\log D n=0.215+\log \left[\left(1-\frac{a_{c}}{P G A}\right)^{2.341}\left(\frac{a_{c}}{P G A}\right)^{-1.438}\right] \pm 0.510
$$


La PGA es la máxima amplitud de la aceleración registrada en el acelerograma en un sitio durante un sismo en particular. Para este estudio se tomó la PGA del estudio de microzonificación sísmica detallada del municipio de Barbosa [18], de donde se tomaron dos escenarios, uno considerado el evento más crítico que coincide con sismofuentes cercanas y gran magnitud, y un segundo escenario de menor criticidad que corresponde con sismofuentes lejanas y menor magnitud (Tabla 3).

Tabla 3. Sismofuentes evaluadas y su PGA correspondiente

\begin{tabular}{|c|c|c|c|}
\hline \multirow{2}{*}{$\begin{array}{l}\text { Sismofuente cercana } \\
\text { Don Matías - Calderas }\end{array}$} & \multicolumn{3}{|c|}{ Sismofuente lejana } \\
\hline & 0.73 & SL7 & 0.21 \\
\hline
\end{tabular}

Fuente: [18]

Para la parametrización de las propiedades geomecánicas de los suelos que conforman el área rural del municipio de Barbosa (Tabla 4), se utilizó la zonificación geotécnica elaborada en los estudios de microzonificación sísmica a partir del mapa geológico del municipio a escala 1:10.000 (ver Figura 2), donde se realizaron perforaciones y ensayos de laboratorio con correlaciones de ensayos de penetración estándar [7], [18]. Para la pendiente, en las zonas rurales, se utilizó el modelo digital de elevación con resolución espacial de 5 m elaborado por el Instituto Geográfico Agustín Codazzi (IGAC) en el proyecto CartoAntioquia.

Tabla 4. Parametrización geotécnica

\begin{tabular}{|c|c|c|c|c|}
\hline Unidad & $\varphi\left(^{\circ}\right)$ & $\mathrm{C}(\mathrm{kPa})$ & $(\mathrm{kN} / \mathrm{m})$ & $\mathrm{k}(\mathrm{m} / \mathrm{s})$ \\
\hline Batolito Antioqueño & 26 & 10 & 17,9 & $1 e-07$ \\
\hline Depósito aluvial & 22,5 & 5 & 19 & $5 e-05$ \\
\hline $\begin{array}{l}\text { Depósito } \\
\text { aluviotorrencial }\end{array}$ & 32 & 5 & 19 & $5 e-05$ \\
\hline $\begin{array}{l}\text { Depósito de } \\
\text { deslizamiento reciente }\end{array}$ & 19 & 10 & 14,9 & $1 e-06$ \\
\hline Llenos antrópicos & 30 & 7,9 & 19 & 0 \\
\hline $\begin{array}{l}\text { Depósito de vertiente } \\
\text { II }\end{array}$ & 22 & 15 & 19,5 & $1 e-06$ \\
\hline $\begin{array}{l}\text { Depósito de vertiente } \\
\text { III }\end{array}$ & 20,5 & 13 & 19,5 & 1e-06 \\
\hline $\begin{array}{l}\text { Depósito de vertiente } \\
\text { IV }\end{array}$ & 20 & 12 & 19,5 & $1 e-06$ \\
\hline $\begin{array}{l}\text { Esquistos de } \\
\text { Cajamarca }\end{array}$ & 18,5 & 20 & 18,2 & $1 e-06$ \\
\hline
\end{tabular}

Fuente: [18].

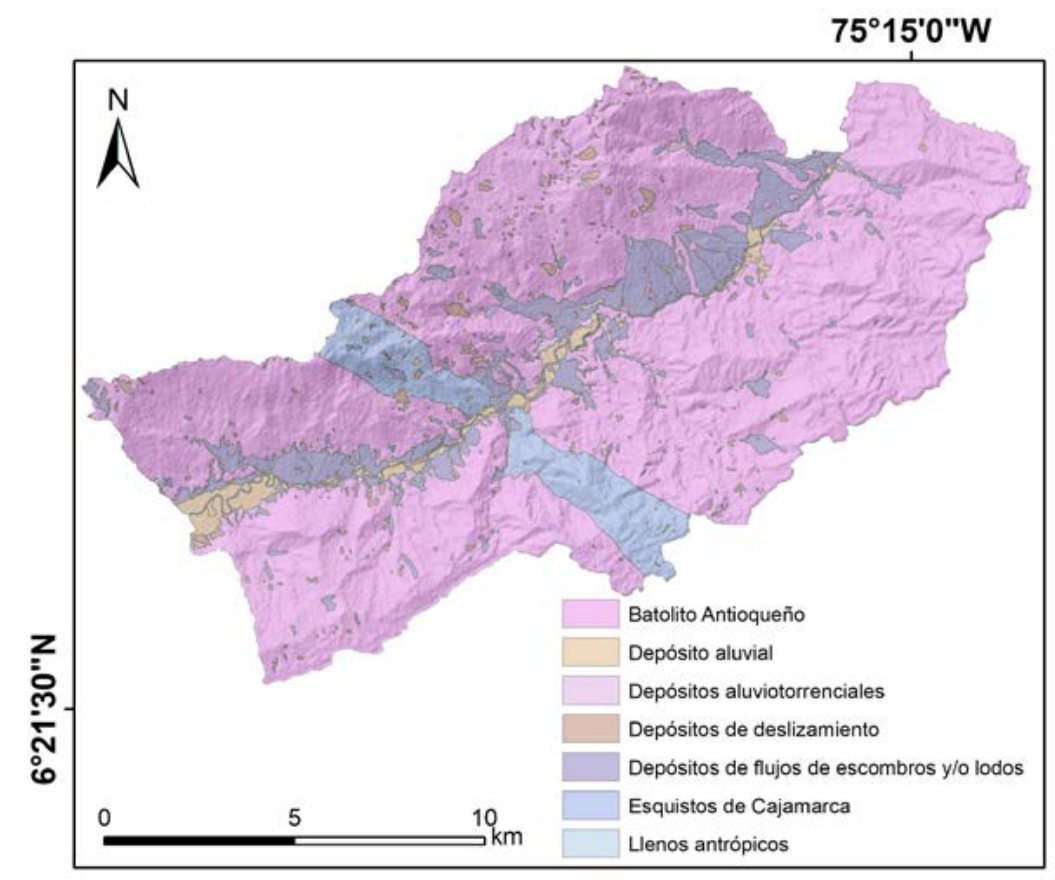

Figura 2. Mapa geológico del municipio de Barbosa.

Fuente: elaboración propia a partir de [18]. 


\section{Resultados}

Como se mencionó anteriormente, para el área urbana del municipio se utilizó el análisis seudoestático con escala de detalle 1:2.000, y para las zonas rurales con escala de detalle 1:5.000 se utilizó el método de Newmark. A continuación, se describen los resultados para cada área de acuerdo con el método implementado.

\section{Área urbana con análisis seudoestático}

Las Figuras 3 y 4 presentan la distribución espacial del Factor de Seguridad (fs) clasificado en tres categorías de amenaza. Se analizaron dos escenarios de saturación del terreno para el mismo evento sísmico: en condiciones saturadas (Figura 3) y en condiciones secas (Figura 4). Las celdas con Fs menor a 1 se consideran inestables, es decir, en amenaza alta, con Fs entre 1 y 1,5 en amenaza media, y con Fs $>1,5$ en amenaza baja, es decir estables.

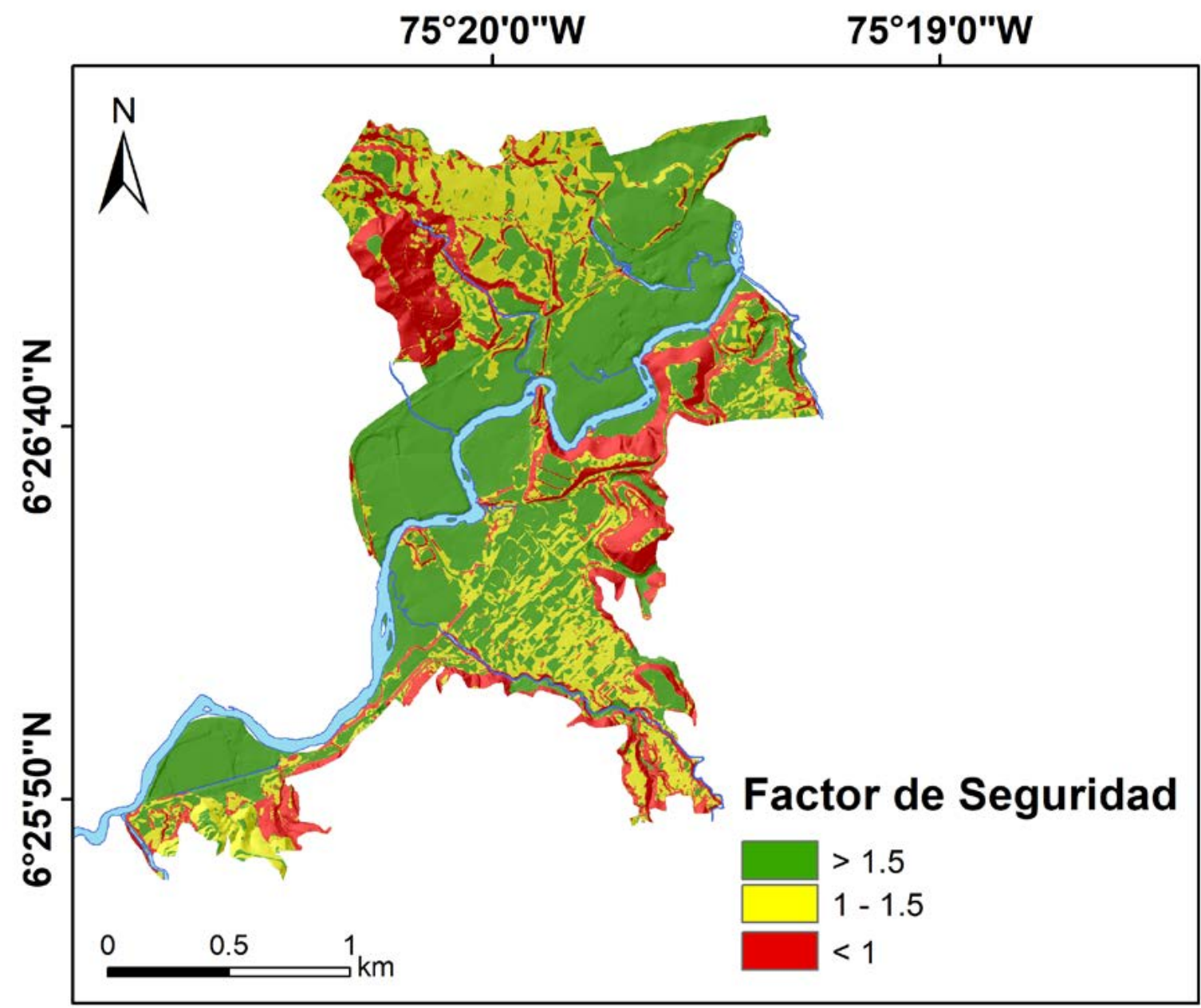

Figura 3. Factor de seguridad seudoestático en condiciones saturadas.

Fuente: elaboración propia. 
Tabla 5. Distribución porcentual de áreas en las categorías de amenaza para el método seudoestático

\begin{tabular}{|c|c|c|}
\hline $\begin{array}{l}\text { Factor de } \\
\text { seguridad }\end{array}$ & $\begin{array}{l}\text { Condiciones } \\
\text { saturadas }\end{array}$ & Condiciones secas \\
\hline$>1,5$ & $49 \%$ & $79 \%$ \\
\hline $1-1,5$ & $29 \%$ & $13 \%$ \\
\hline$<1$ & $22 \%$ & $8 \%$ \\
\hline
\end{tabular}

Fuente: elaboración propia.

Como se observa en la Tabla 5, en condiciones saturadas el $22 \%$ del territorio se encuentra en amenaza alta, con un Fs menor a 1 . En amenaza media se encuentra el $29 \%$, y el $49 \%$ restante tiene un Fs mayor a 1,5. Para las condiciones secas, solo el $8 \%$ del territorio se encuentra en amenaza alta y el área con Fs mayor a 1,5 aumenta hasta el 79\%, dejando la amenaza media con un $13 \%$. A partir de la comparación de los resultados entre los dos escenarios de agua en el suelo, se observa que los Fs mayores a 1,5 disminuyen el $30 \%$ en condiciones saturadas. De la misma manera, los valores menores a 1 , y los que están entre 1 y 1,5 , aumentan $13 \%$ y $16 \%$, respectivamente.

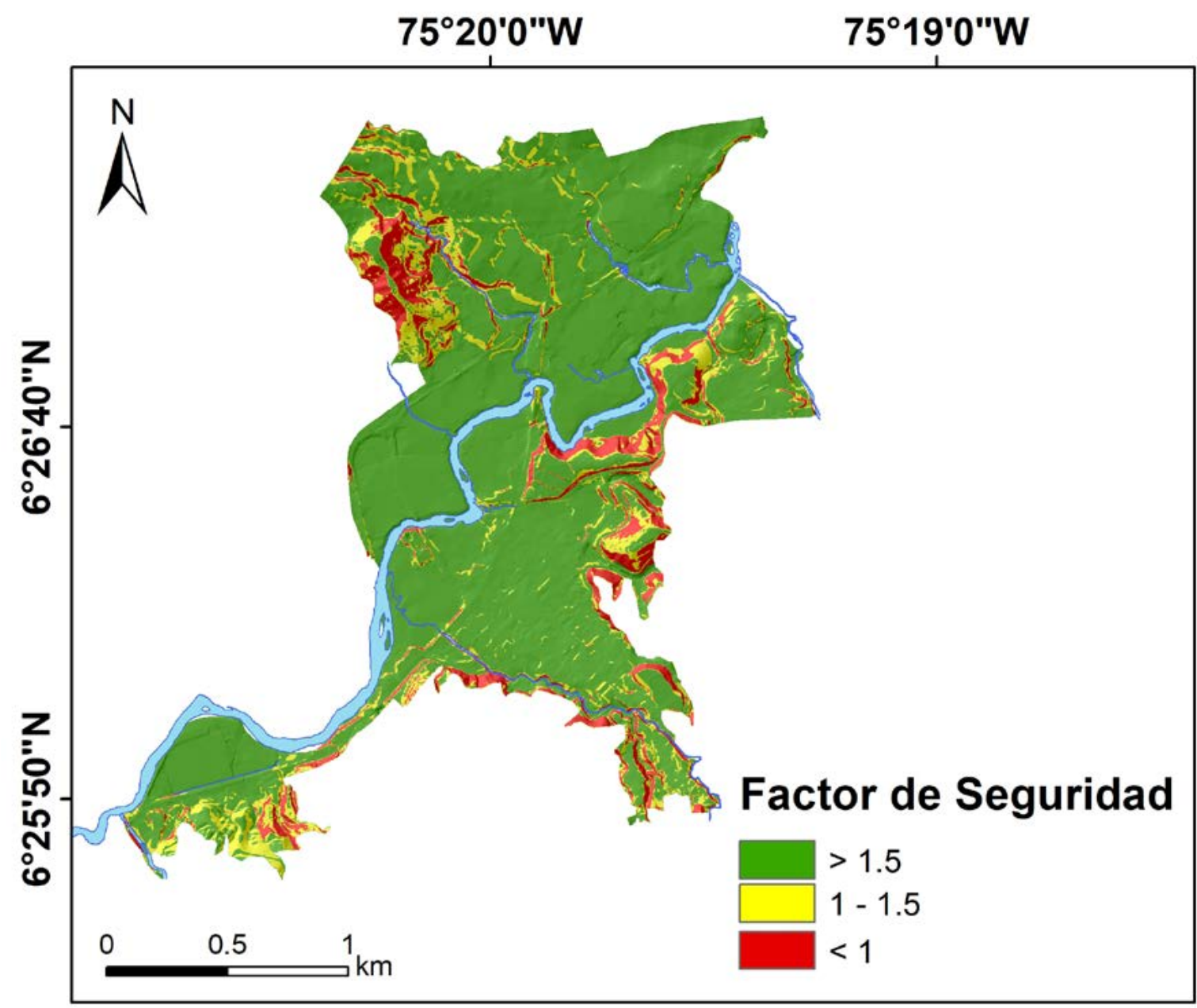

Figura 4. Factor de seguridad seudoestático en condiciones secas.

Fuente: elaboración propia. 
La inestabilidad en el método seudoestático señala un control de la geometría de la ladera, específicamente por la pendiente. En condiciones saturadas se enmarca en las altas pendientes como en algunas márgenes de las quebradas y en los lomos que generan las rocas en la parte baja. Las zonas de baja pendiente se encuentran en amenaza baja con valores de Fs mayores a 1,5. En condiciones secas del terreno, las celdas con Fs menores a 1 son muy pocas y se restringen a las áreas de mayor pendiente. Las celdas estables $(\mathrm{FS}<1,5)$ dominan el área, mientras que las zonas en amenaza media, como en condiciones saturadas, se concentran en las márgenes de las quebradas y en las pendientes moderadas asociadas a los lomos.

En cuanto a la geología, la inestabilidad se asocia a los suelos graníticos del Batolito Antioqueño que se encuentran en las zonas que limitan el área urbana, mientras que los depósitos, por su baja pendiente, se caracterizan por ser estables en condiciones secas, aunque alcanzan amenaza media en condiciones saturadas.

\section{Área rural con análisis de desplazamientos permanentes}

Los escenarios modelados con el método de Newmark consideran las condiciones de saturación del terreno y la distancia de la sismofuente. Las Figuras 4 a 7 presentan los escenarios modelados a partir de los dos eventos sísmicos, cada uno bajo condiciones saturadas (escenario 1) y secas (escenario 2). Las Figuras 4 y 6 corresponden a la sismofuente cercana en condiciones saturadas y condiciones secas, respectivamente, mientras que las Figuras $5 \mathrm{y}$ 7 corresponden al escenario de sismofuente lejana en condiciones saturadas (escenario 3) y condiciones secas (escenario 4), respectivamente. Estos resultados se clasifican de acuerdo con las categorías de amenaza propuestas por Jibson y Michael [53].

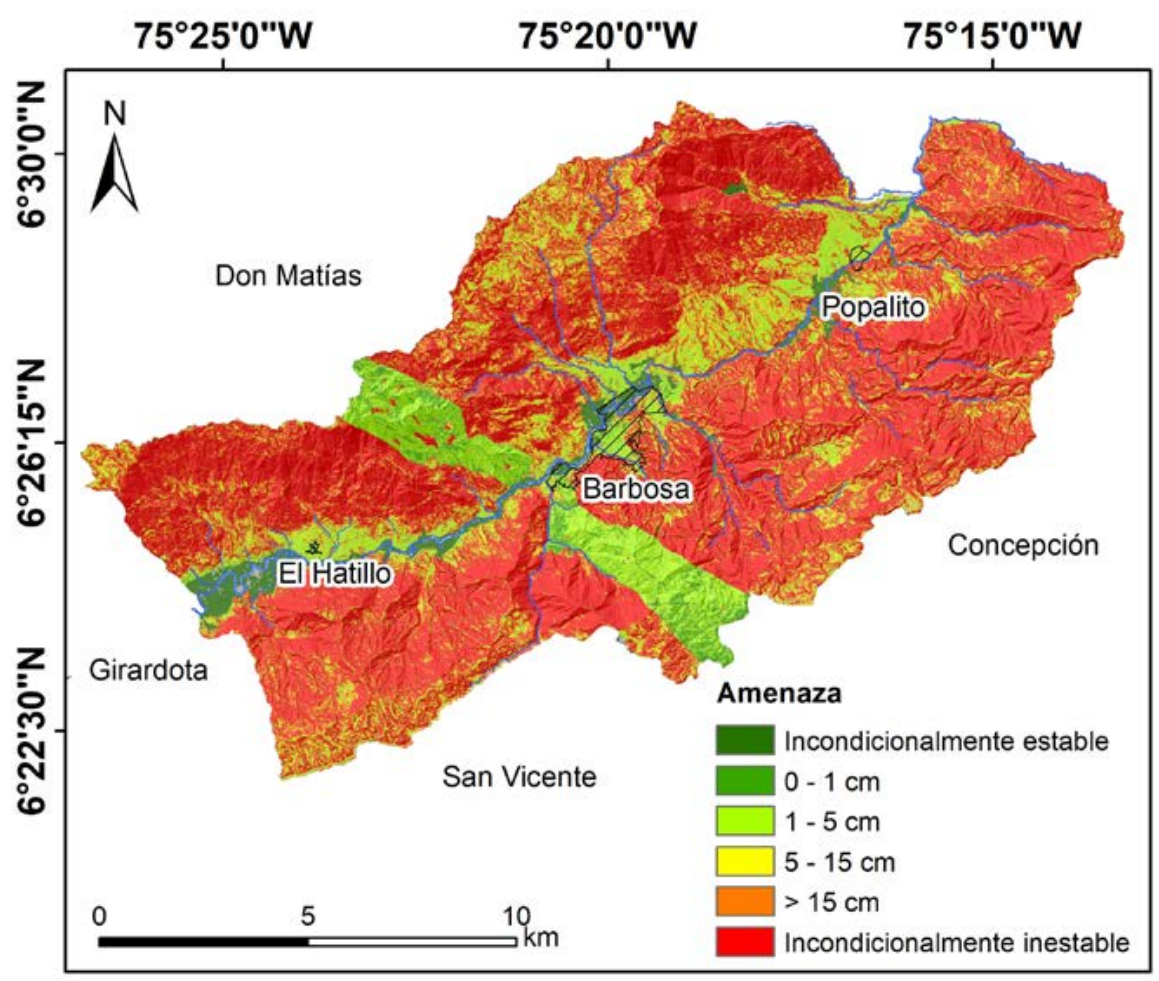

Figura 5. Amenaza de movimiento en masa detonado por sismos para el escenario de sismofuente cercana y condiciones saturadas (escenario 1).

Fuente: elaboración propia. 


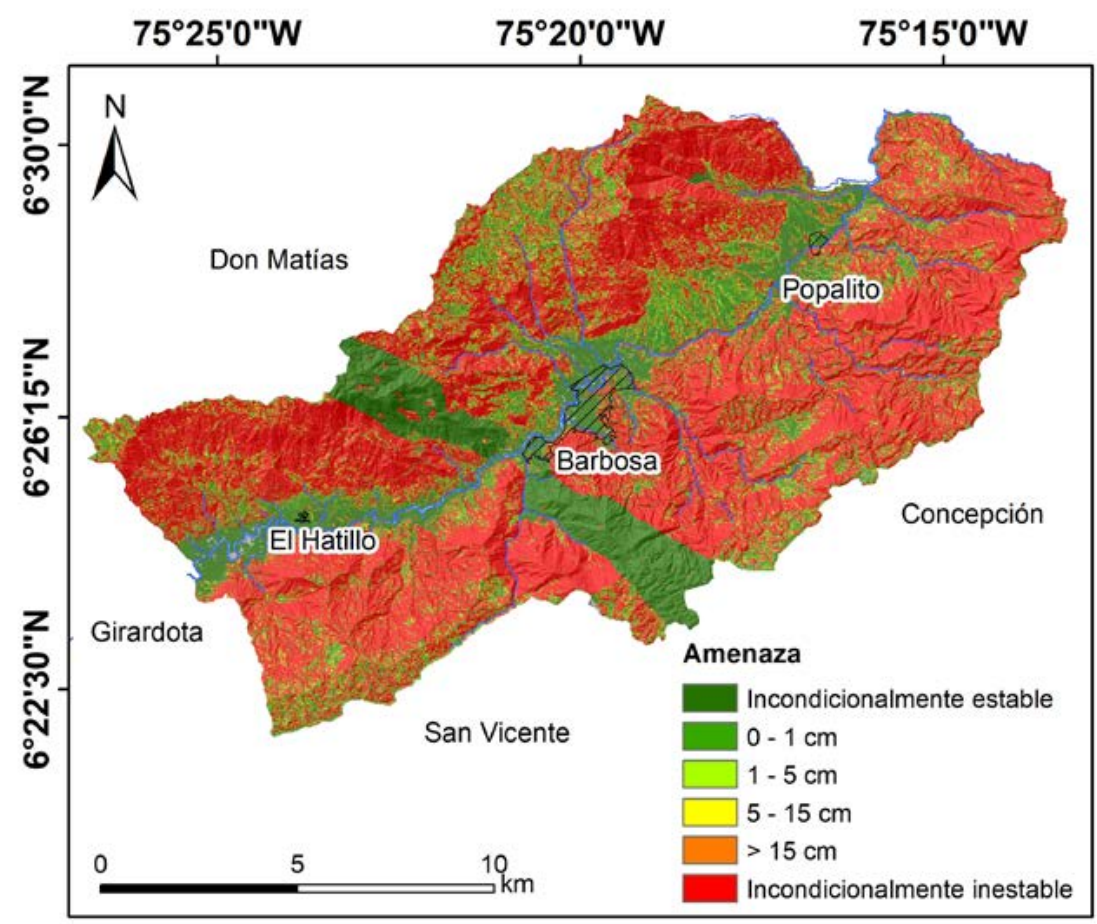

Figura 6. Amenaza de movimiento en masa detonado por sismos para el escenario de sismofuente lejana y condiciones saturadas (escenario 2).

Fuente: elaboración propia.

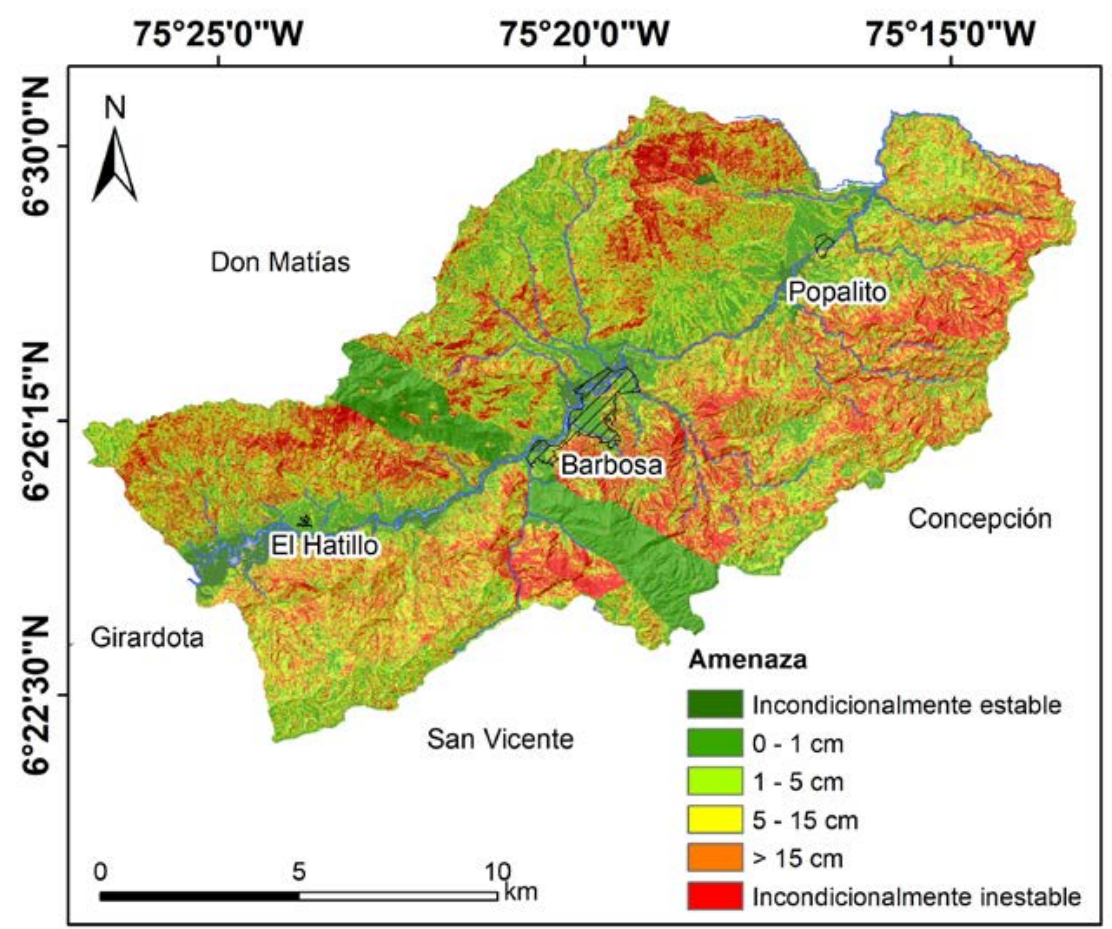

Figura 7. Amenaza de movimiento en masa detonado por sismos para el escenario de sismofuente cercana y condiciones secas (escenario 3).

Fuente: elaboración propia. 


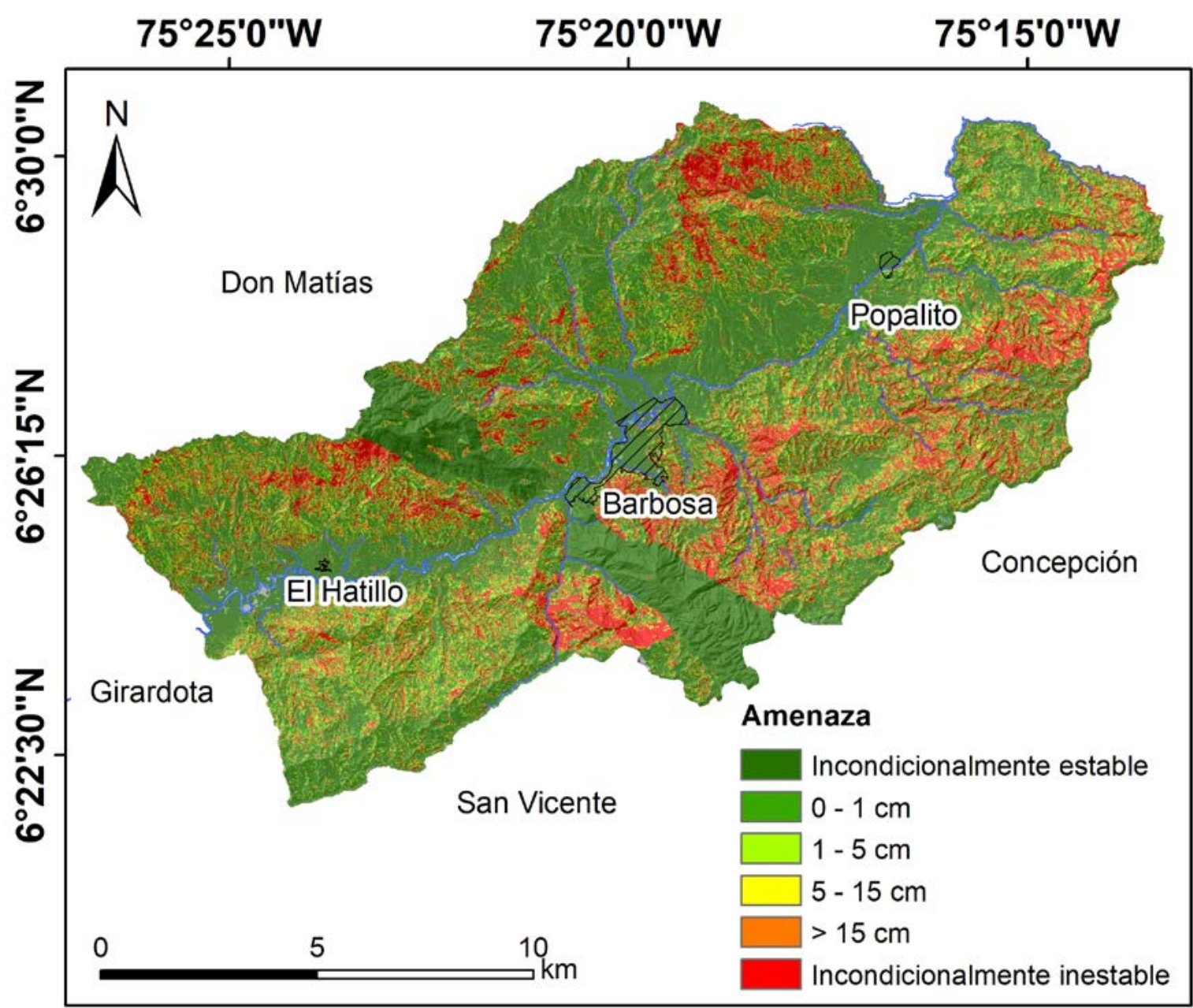

Figura 8. Amenaza de movimiento en masa detonados por sismos para el escenario de sismofuente lejana y condiciones secas (escenario 4).

Fuente: elaboración propia.

Las diferentes categorías de la amenaza sísmica en los escenarios analizados son controladas principalmente por las unidades geológicas superficiales, aunque también se presenta una relación directamente proporcional con la pendiente.

Como se observa en la Tabla 4, los resultados en el escenario de sismofuente cercana en condiciones saturadas corresponden al escenario más crítico (Figura 4), debido a tener un sismo con mayor PGA y suelos en condiciones saturadas que reducen los esfuerzos efectivos y la resistencia al corte del suelo, mientras que el escenario de sismofuente lejana y condiciones secas corresponde al escenario de menor criticidad (Figura 7).
Tabla 6 . Distribución porcentual de áreas en las categorías de amenaza para el método de Newmark

\begin{tabular}{|c|c|c|c|c|}
\hline & \multicolumn{4}{|c|}{ Escenario } \\
\hline Categorías & 1 & 2 & 3 & 4 \\
\hline Estable & $3 \%$ & $17 \%$ & $3 \%$ & $46 \%$ \\
\hline $0-1 \mathrm{~cm}$ & $2 \%$ & $11 \%$ & $23 \%$ & $19 \%$ \\
\hline $1-5 \mathrm{~cm}$ & $13 \%$ & $5 \%$ & $21 \%$ & $8 \%$ \\
\hline $5-15 \mathrm{~cm}$ & $8 \%$ & $3 \%$ & $15 \%$ & $4 \%$ \\
\hline$>15 \mathrm{~cm}$ & $16 \%$ & $5 \%$ & $21 \%$ & $6 \%$ \\
\hline Inestable & $59 \%$ & $59 \%$ & $17 \%$ & $17 \%$ \\
\hline
\end{tabular}

Fuente: elaboración propia. 
La Tabla 6 presenta los resultados en términos de porcentaje para cada uno de los escenarios evaluados. Clasificado como "inestable" se encuentra el $59 \%$ del municipio en los escenarios 1 y 2 y el $17 \%$ en los escenarios 3 y 4 . Es decir, al pasar de condiciones secas a saturadas, las celdas que se caracterizan por una inestabilidad intrínseca del suelo aumentan $42 \%$. En cambio, dentro de la categoría "estable" se puede ver el contraste entre los efectos de los dos sismos, ya que en condiciones saturadas aumentan estas celdas estables un 14\% y en condiciones secas un $43 \%$, al pasar del sismo más fuerte y/o más cercano al más lejano y/o de menor magnitud.

La distribución de las celdas en las categorías observadas en la Tabla 6 señala la criticidad de cada escenario analizado. El escenario 1, que corresponde al escenario más crítico, muestra un dominio de las celdas inestables y de grandes desplazamientos $(>15 \mathrm{~cm})$ con un $75 \%$, y con solo un $3 \%$ de celdas estables. Por el contrario, al analizar los resultados del escenario 4, que se podría ver como el escenario menos crítico, el dominio es de las celdas estables con un $46 \%$ y de celdas con desplazamientos menores a $1 \mathrm{~cm}$ con 19\%, mientras que las que experimentan desplazamientos mayores a $5 \mathrm{~cm}$ y las inestables presentan un porcentaje mucho menor, de $27 \%$ en conjunto. En los escenarios intermedios 2 y 3 , se marca la influencia de la saturación en la estabilidad, ya que las celdas inestables aumentan $42 \%$ al evaluar el sismo mayor en condiciones secas y el menor en saturadas; también la influencia del evento sísmico se marca en esta comparación al aumentar 14\% las celdas estables. En el escenario 2 la mayor concentración de área está en categoría inestable, mientras que en el 3 se distribuyen muy homogéneamente en los desplazamientos, es decir, no son incondicionalmente estables ni inestables.

\section{Análisis y conclusiones}

Los procesos de ordenación del territorio, los cuales obligatoriamente deben realizar los municipios de acuerdo con la Ley 388 de 1997, involucran como elemento fundamental dentro de su análisis la evaluación de la amenaza por fenómenos de origen natural. Colombia, como resultado del proceso de subducción, presenta terrenos montañosos y tectónicamente activos con la ocurrencia regular de sismos de magnitudes y profundidades diversas. $\mathrm{Y}$ aunque el principal agente detonante de los movimientos en masa en nuestro país es la lluvia, los sismos tienen una gran capacidad de generar un enjambre de movimientos en masa con volúmenes considerables y gran capacidad destructiva en los centros urbanos. En este sentido, el Decreto 1077 de 2015, que regula en Colombia la elaboración de los estudios de amenaza para los planes de ordenamiento territorial, exige como parte del análisis de la amenaza por movimientos en masa considerar no solamente la lluvia como factor detonante, sino además los sismos.

De acuerdo con Jibson [39] y Keefer [3], los sismos con $\mathrm{ML}>4,0$ tienen la capacidad de generar movimientos en masa, tipo caídas y deslizamientos en laderas susceptibles; mientras que sismos con $\mathrm{ML}>6,0$ tienen la capacidad de generar movimientos en masa sobre extensas áreas, tipo caídas y deslizamientos profundos principalmente. De acuerdo con la sismicidad histórica registrada en el Valle de Aburrá y los estudios de microzonificación sísmica del mismo, sismos con magnitudes intermedias han afectado el Valle de Aburrá, y es probable la ocurrencia de sismos de mayor magnitud con consecuencias considerables, como resultado de la acelerada ocupación de las laderas del Valle durante las últimas décadas [18]. Para el municipio de Barbosa, los estudios de microzonificación sísmica señalan sismofuentes a distancias entre $2,5 \mathrm{~km}$ y $72 \mathrm{~km}$ con magnitudes entre 6,8 y 7,7 y valores de PGA de 0,73 y 0,21 respectivamente [18]; esto, de acuerdo con Jibson [39] y Keefer [3], significa la posibilidad de movimientos en masa profundos y enjambres de movimientos.

Como se describe en el presente trabajo, la implementación de los métodos Newmark y seudoestático permite evaluar la amenaza de movimientos en masa detonados por sismo. Las metodologías tienen en consideración las propiedades geomecánicas del suelo, las condiciones sísmicas locales y los efectos en superficie de las cargas sísmicas producto de fuentes sismogénicas cercanas y lejanas, permitiendo estimar el comportamiento dinámico de las laderas ante la ocurrencia de un sismo. 
Los resultados obtenidos concuerdan con resultados alrededor del mundo [10], [54], [55], donde la saturación del terreno influye negativamente en la estabilidad de las laderas en condiciones dinámicas. Para la ciudad de Medellín en el Valle de Aburrá, Vega Gutiérrez [2], considerando el sismo como factor detonante, encuentra una importante caída en la estabilidad de las laderas de acuerdo con el aumento del nivel freático. Para el municipio de Barbosa, la respuesta dinámica de los suelos en condiciones saturadas presenta un aumento del $42 \%$ de las celdas inestables en comparación con las condiciones secas.

La implementación del efecto sísmico en el modelo de equilibrio límite para talud infinito, utilizado en ambos modelos, permite evaluar la influencia de las condiciones de saturación y cargas sísmicas de manera simultánea, como eventos detonantes de los movimientos en masa. Esto se convierte en una ventaja para el análisis, ya que permite evaluar la condición más crítica asociada a un sismo de gran magnitud bajo condiciones saturadas. Para el presente trabajo, y en ambas metodologías, cuando se supuso una condición de saturación de las laderas tanto en la zona urbana como en la rural, se presentó un incremento de las áreas de amenaza alta con respecto a la condición seca, lo que implica que el efecto de las fuerzas sísmicas sería más crítico en épocas de lluvias qué en épocas secas, lo cual va en la misma dirección de otros trabajos realizados en el Valle de Aburrá [43], [44].

Adicionalmente, tanto para el análisis seudoestático como por desplazamientos permanentes, se aprecia que la estabilidad asociada al detonante sismo está también altamente relacionada con la pendiente y la geología. Las zonas de baja pendiente tienden a ser incondicionalmente estables, mientras que las de mayor pendiente tienden a ser inestables aún en el escenario menos crítico. En este mismo sentido, Montoya y Gutiérrez [44] encuentran para el Valle de Aburrá un aumento en las probabilidades de falla por sismo en laderas con pendientes superiores al $40 \%$. En cuanto a la geología del área de estudio, la estabilidad se asocia principalmente a las unidades graníticas como el Batolito Antioqueño, debido a la poca cohesión de los suelos que desarrollan.
Respecto al análisis seudoestático, en la presente investigación se obtiene el coeficiente horizontal seudoestático $\left(k_{h}\right)$ mediante la metodología descrita en la NSR-10 [50], la cual tiene en cuenta la aceleración pico efectiva del terreno $\left(A_{a}\right)$. En efecto, el valor de considera el coeficiente de ampliación $\left(F_{a}\right)$, con el fin de evaluar el efecto sísmico para periodos cortos según el perfil de suelo, clasificado según la velocidad de onda cortante como principal criterio. Además, incluye el coeficiente de importancia (I) como medida de la variabilidad de las estructuras expuestas. Sin embargo, en esta investigación se optó por utilizar el valor unitario, debido a la poca información del uso en la infraestructura de la zona de estudio. Por lo tanto, la metodología implementada para obtener el coeficiente horizontal seudoestático, tiene en consideración las características locales del sitio en la evaluación del efecto sísmico. Cabe destacar que el método seudoestático plantea el efecto del sismo bajo condiciones permanentes y en dirección horizontal, de manera que los resultados deben ser empleados como una aproximación al comportamiento del suelo ante un evento sísmico.

Las metodologías presentadas muestran un gran potencial como herramienta de análisis de la amenaza de movimientos en masa detonados por sismos en zonas de montaña, las cuales son áreas importantes y altamente pobladas en los Andes Colombianos. Sin embargo, es importante resaltar que considerando la variabilidad espacial de los parámetros del suelo y los modelos simplificados que utilizan los métodos presentados, los resultados obtenidos deben ser evaluados como de carácter regional para identificar áreas críticas a priorizar. Es necesaria, por lo tanto, y de acuerdo con lo que especifica el Decreto 1077 de 2015, la elaboración de estudios de detalle en las áreas críticas identificadas, donde se realice una exhaustiva exploración directa y ensayos de laboratorio que permitan reducir los niveles de incertidumbre asociados a los parámetros del suelo, e implementar modelos de deformación para mayor detalle en laderas específicas.

Adicionalmente al carácter regional del presente estudio, es importante tener en cuenta que los escenarios modelados buscan solo evaluar los 
escenarios extremos ante un sismo del municipio de Barbosa, y no representan los escenarios más probables, ya que se encuentran fuera del alcance del presente estudio. Estudios recientes han estimado para el Valle de Aburrá las probabilidades de falla entre $3 \times 10-4$ y $2 \times 10-3$ para un sismo con una probabilidad de excedencia del $10 \%$ en 50 años [44].

\section{Agradecimientos}

Este estudio fue posible gracias a la financiación del Área Metropolitana del Valle de Aburrá, en convenio con la Universidad Nacional de Colombia-Medellín.

\section{Referencias}

[1] R. W. Jibson, E. L. Harp y J. A. Michael, "A Method for Producing Digital Probabilistic Seismic Landslide Hazard Maps", Eng. Geol., vol. 58, n. ${ }^{\circ}$ 3-4, pp. 271-289, dic. 2000. Dor: https://doi.org/10.1016/S00137952(00)00039-9

[2] J. A. Vega, Estimación del riesgo por deslizamientos de laderas generados por eventos sísmicos en la ciudad de Medellín usando herramientas de la geomática-Caso aplicado a edificaciones urbanas, tesis MA, Facultad de Ingeniería y Ciencias Astronómicas y Geofísicas, Universidad Nacional de La Plata, Argentina, 2013.

[3] T. L. Youd, "Landslides Caused by Earthquakes: Discussion", Bull. Geol. Soc. Am., vol. 96, n. ${ }^{\circ}$ 8, pp. 1091-1092, 1985. DoI: https://doi.org/10.1130/0016-7606(1985)96<1091:LCBEDA>2.0. $\mathrm{CO} ; 2$

[4] E. L. Harp y R. W. Jibson, "Landslides Triggered by the 1994 Northridge, California, Earthquake", Bull. Seismol. Soc. Am., vol. 86, n. ${ }^{\circ}$ 1, Supl. B, pp. S319-S332, 1996. Dor: https://doi.org/10.3133/ofr95213

[5] O. Sánchez y E. Aristizábal, "Spatial and Temporal Paterns and Socieconomic Impact of Landslides in Colombia", Poster Eur. Geosci. Meet. Austria 8-13 April, n. ${ }^{\circ}$ June, 2018. Disponible en https://ui.adsabs.harvard.edu/abs/2018EGUGA..20.3575S/abstract

[6] J. M. Martínez, R. L. Schuster, T. J. Casadevall y K. M. Scott, "Landslides and Debris Flows Triggered by the 6 June 1994 Paez Earthquake, Southwestern Colombia", Landslide News, vol. 9, pp. 13-15, 1995.

[7] Universidad de los Andes, Armonización de la microzonificación sísmica de los municipios del Valle de Aburrá, e inclusión de los corregimientos de Medellín [en línea]. Disponible en https://repositorio.gestiondelriesgo.gov.co/handle/20.500.11762/19862

[8] C. García, "Estado del donocimiento de los depósitos de vertiente del Valle de Aburrá", Boletín Ciencias la Tierra, vol. 19, pp. 101-112, 2006.

[9] E. Aristizábal y S. Yokota, “Evolución geomorfológica del Valle de Aburrá y sus implicaciones en la ocurrencia de movimientos en masa", Boletín Ciencias de la Tierra, vol. 24, pp. 5-18, 2008. Disponible en http:// www.scielo.org.co/scielo.php?script $=$ sci_arttext\&pi$\mathrm{d}=$ S0120-36302008000300002\&lng=en\&tlng=es

[10] B. B. Mirus, R. E. Becker, R. L. Baum y J. B. Smith, "Integrating Real-Time Subsurface Hydrologic Monitoring with Empirical Rainfall Thresholds to Improve Landslide Early Warning", Landslides, vol. 15, n. ${ }^{\circ}$ 10, pp. 1909-1919, 2018. DoI: https://doi.org/10.1007/ s10346-018-0995-Z

[11] M. Hermelin, D. A. Rendón y G. E. Toro, "Modelo cronoestratigráfico para el emplazamiento de los depósitos de vertiente en el Valle de Aburrá”, Boletín Ciencias la Tierra, vol. 18, n. ${ }^{\circ} 18$, pp. 103-118, 2006.

[12] F. Cediel, R. P. Shaw y C. Cáceres, "Tectonic Assembly of the Northern Andean Block", AAPG Mem., n. ${ }^{\circ} 79$, pp. 149-152, 2005.

[13] F. Cediel y R. P. Shaw, Geology and Tectonics of Northwestern South America, Suiza: Springer, 2019. DOI: https://doi.org/10.1007/978-3-319-76132-9

[14] J. N. Kellogg, V. Vega, T. C. Stailings y C. L. V. Aiken, “Tectonic Development of Panamá, Costa Rica, and the Colombian Andes: Constraints from Global Positioning System Geodetic Studies and Gravity", Spec. Pap. Geol. Soc. Am., vol. 295, pp. 75-90, 1995. DoI: https://doi.org/10.1130/SPE295-p75

[15] A. Taboada et al., "Geodynamics of the northern Andes: Subductions and intracontinental deformation (Colombia)", Tectonics, vol. 19, n. ${ }^{\circ}$ 5, pp. 787-813, 2000. DOI: https://doi.org/10.1029/2000TC900004

[16] R. Trenkamp, J. N. Kellogg, J. T. Freymueller y H. P. Mora, "Wide Plate Margin Deformation, Southern Central América and Northwestern South América, CASA GPS observations", J. South Am. Earth Sci., vol. 15, n. ${ }^{\circ}$ 2, pp. 157-171, 2002. DoI: https://doi.org/10.1016/ S0895-9811(02)00018-4

[17] S. Noriega, Geomorfología tectónica del noroccidente de la Cordillera Central, Andes del Norte - Colombia, tesis ma, Facultad de Minas, Departamento de Minas y Materiales, Universidad Nacional de Colombia, Medellín, 2016. 
[18] Unidad Nacional para la Gestión del Riesgo de Desastres, "Microzonificación sísmica detallada de los municipios de Barbosa, Girardota, Copacabana, Sabaneta, La Estrella, Caldas y Envigado, Informe Final”, Área Metrop. del Val. Aburrá, Consorcio Microzonificación, 2006. Disponible en https://repositorio.gestiondelriesgo.gov.co/handle/20.500.11762/19862

[19] O. M. Mariño y J. Duque-Trujillo, Caracterización de La cinemática y cálculo de paleo-tensores de esfuerzo para el conjunto principal de fallas en el Valle de Aburrá, Implicaciones Tectónicas, tesis BA, Departamento de Ciencias de la Tierra, Universidad EAfIT, Medellín, 2017. Disponible en https://repository.eafit.edu.co/ handle/10784/12412

[20] J. I. Barredo, A. Benavides, J. Hervás y C. J. Van Westen, "Comparing Heuristic Landslide Hazard Assessment Techniques Using gis in the Tirajana Basin, Gran Canaria Island, Spain", Int. J. Appl. Earth Obs. Geoinf., vol. 2000, n. ${ }^{\circ}$ 1, pp. 9-23, 2000. Dor: https:// doi.org/10.1016/S0303-2434(00)85022-9

[21] L. Ayalew, H. Yamagishi, H. Marui y T. Kanno, "Landslides in Sado Island of Japan: Part II. gis-Based Susceptibility Mapping with Comparisons of Results from two Methods and Verifications", Eng. Geol., vol. 81, n. ${ }^{\circ}$ 4, pp. 432-445, 2005. DoI: https://doi.org/10.1016/j.enggeo.2005.08.004

[22] A. Carrara, M. Cardinali, R. Detti, F. Guzzetti, V. Pasqui y P. Reichenbach, "gis Techniques and Statistical Models in Evaluating Landslide Hazard", Earth Surf. Process. Landforms, vol. 16, n. ${ }^{\circ}$ 5, pp. 427-445, 1991. DOI: https://doi.org/10.1002/esp.3290160505

[23] H. Hong, B. Pradhan, C. Xu y D. Tien Bui, "Spatial Prediction of Landslide Hazard at the Yihuang Area (China) Using Two-class Kernel Logistic Regression, Alternating Decision Tree and Support Vector Machines", Catena, vol. 133, pp. 266-281, 2015. Dor: https:// doi.org/10.1016/j.catena.2015.05.019

[24] W. M. Abdulwahid y B. Pradhan, "Landslide Vulnerability and Risk Assessment for Multi-Hazard Scenarios Using Airborne Laser Scanning Data (LIDAR)", Landslides, vol. 14, n. 3 , pp. 1057-1076, 2017. Dor: https://doi.org/10.1007/s10346-016-0744-0

[25] G. Demir, M. Aytekin y A. Akgun, "Landslide Susceptibility Mapping by Frequency Ratio and Logistic Regression Methods: An Example from Niksar-Resadiye (Tokat, Turkey)", Arab. J. Geosci., vol. 8, n. ${ }^{\circ}$ 3, pp. 1801-1812, 2015. DOI: https://doi.org/10.1007/s12517$014-1332-\mathrm{Z}$
[26] C. J. Van Westen y M. T. J. Terlien, “An Approach towards Deterministic Landslide Hazard Analysis in gis. A Case Study from Manizales (Colombia)", Earth Surf. Process. Landforms, vol. 21, n. ${ }^{\circ}$ 9, pp. 853-868, 1996. DoI: https://doi.org/10.1002/(SICI)1096-9837(19 9609)21:9<853::AID-ESP676>3.0.CO;2-C

[27]D. R. Montgomery y W.E. Dietrich, “A Physically Based Model for the Topographic Control on Shallow Landsliding”, Water Resour. Res., vol. 30, n. ${ }^{\circ}$, pp. 1153-1171, 1994. Dor: https://doi.org/10.1029/93WR02979

[28] E. Aristizábal, J. I. Vélez, H. E. Martínez y M. Jaboyedoff, "shia Landslide: a Distributed Conceptual and Physically Based Model to Forecast the Temporal and Spatial Occurrence of Shallow Landslides Triggered by Rainfall in Tropical and Mountainous Basins", Landslides, vol. 13, n. ${ }^{\circ}$, pp. 497-517, 2016. DoI: https://doi. org/10.1007/s10346-015-0580-7

[29] L. N. Thanh y F. De Smedt, "Slope Stability Analysis Using a Physically Based Model: A Case Study from A Luoi District in Thua Thien-Hue Province, Vietnam", Landslides, vol. 11, n. ${ }^{\circ}$ 5, pp. 897-907, 2014. DoI: https:// doi.org/10.1007/s10346-013-0437-x

[30] R. W. Jibson, "Methods for assessing the stability of slopes during earthquakes - A retrospective", Eng. Geol., vol. 122, n. ${ }^{\circ} 1-2$, pp. 43-50, 2011. DoI: https://doi. org/10.1016/j.enggeo.2010.09.017

[31] K. Terzaghi, "Mechanism of Landslides", en Application of Geology to Engineering Practice. Geological Society of America, 2015, pp. 83-123. DoI: https://doi. org/10.1130/Berkey.1950.83

[32] C. Melo y S. Sharma, "Seismic Coefficients for Pseudostatic Slope Analysis", 13th World Conf. Earthq. Eng., n. ${ }^{\circ}$ 369, p. 15, 2004.

[33]N. M. Newmark, "Effects of Earthquakes on Dams and Embankments", Geotechnique, vol. 15, n. ${ }^{\circ}$ 2, pp. 139-160, 1965. DoI: https://doi.org/10.1680/ geot.1965.15.2.139

[34] C. Meletti et al., "A Seismic Source Zone Model for the Seismic Hazard Assessment of the Italian Territory", Tectonophysics, vol. 450, n. ${ }^{\circ} 1-4$, pp. 85-108, 2008. DOI: https://doi.org/10.1016/j.tecto.2008.01.003

[35] R. K. McGuire, "Probabilistic Seismic Hazard Analysis and Design Earthquakes: Closing the Loop", Bull. - Seismol. Soc. Am., vol. 85, n. ${ }^{\circ}$ 5, pp. 1275-1284, 1995.

[36] S. C. Bhatia, M. R. Kumar y H. K. Gupta, "A Probabilistic Seismic Hazard Map of India and Adjoining Regions”, Annali di Geofisica, vol. 42, n. ${ }^{\circ}$ 6, pp. 1153 1164, 1999. 
[37] C. T. Lee, C. C. Huang, J. F. Lee, K. L. Pan, M. L. Lin y J. J. Dong, "Statistical Approach to Earthquake-Induced Landslide Susceptibility", Eng. Geol., vol. 100, n. ${ }^{\circ} 1-2$, pp. 43-58, 2008. Dor: https://doi.org/10.1016/j. enggeo.2008.03.004

[38] S. Lee y B. Pradhan, "Probabilistic Landslide Hazards and Risk Mapping on Penang Island, Malaysia", J. Earth Syst. Sci., vol. 115, n. ${ }^{\circ}$ 6, pp. 661-672, 2006. Dor: https://doi.org/10.1007/s12040-006-0004-0

[39] R. W. Jibson, "Predicting Earthquake-Induced Landslide Displacements Using Newmark's Sliding Block Analysis”, Transp. Res. Rec., n. ${ }^{\circ}$ 1411, pp. 9-17, 1993.

[40] V. G. Kossobokov, V. I. Keilis-Borok, D. L. Turcotte y B. D. Malamud, "Implications of a Statistical Physics Approach for Earthquake Hazard Assessment and Forecasting", Pure Appl. Geophys., vol. 157, n. ${ }^{\circ} 11-$ 12, pp. 2323-2349, 2000. DoI: https://doi.org/10.1007/ PL00001086

[41]A. Refice y D. Capolongo, "Probabilistic Modeling of Uncertainties in Earthquake-Induced Landslide Hazard Assessment”, Comput. Geosci., vol. 28, n. ${ }^{\circ}$ 6, pp. 735-749, 2002. DoI: https://doi.org/10.1016/S00983004(01)00104-2

[42] D. Asprone, F. Jalayer, A. Prota y G. Manfredi, "Proposal of a Probabilistic Model for Multi-Hazard Risk Assessment of Structures in Seismic Zones Subjected to Blast for the Limit State of Collapse", Struct. Saf., vol. 32, n. ${ }^{\circ}$ 1, pp. 25-34, 2010. Dor: https://doi.org/10.1016/j. strusafe.2009.04.002

[43] J. A. Vega y C. A. Hidalgo, "Quantitative Risk Assessment of Landslides Triggered by Earthquakes and Rainfall Based on Direct Costs of Urban Buildings", Geomorphology, vol. 273, n. ${ }^{\circ}$ August, pp. 217-235, 2016. DOI: https://doi.org/10.1016/j.geomorph.2016.07.032

[44] C. Hidalgo y J. Vega, "Estimación de la amenaza por deslizamientos detonados por sismos y lluvia (Valle de Aburrá-Colombia)", Rev. eia, vol. 11, n. ${ }^{\circ} 22$, pp. 103$117,2014$.

[45] M. A. Salgado-Gálvez, D. Zuloaga-Romero, G. A. Bernal, M. G. Mora y O. D. Cardona, "Fully Probabilistic Seismic Risk Assessment Considering Local Site Effects for the Portfolio of Buildings in Medellín, Colombia”, Bull. Earthq. Eng., vol. 12, n. ${ }^{\circ}$ 2, pp. 671-695, 2014. DoI: https://doi.org/10.1007/s10518-013-9550-4

[46] D. Giardini, “The Global Seismic Hazard Assessment Program (gshap) - 1992/1999”, Ann. di Geofis., vol. 42, n. ${ }^{\circ}$, pp. 957-974, 1999.
[47] Universidad Nacional de Colombia - Sede Medellín, "Estudios básicos de amenaza por movimientos en masa, inundaciones y avenidas torrenciales en los municipios de Caldas, La Estrella, Envigado, Itagüí, Bello, Copacabana y Barbosa, para la incorporación de la Gestión Del Riesgo En La Planificación Territorial”, p. 350, 2018.

[48] R. Baker, R. Shukha, V. Operstein y S. Frydman, "Stability Charts for Pseudo-Static Slope Stability Analysis", Soil Dyn. Earthq. Eng., vol. 26, n. ${ }^{\circ}$ 9, pp. 813-823, 2006. DoI: https://doi.org/10.1016/j.soildyn.2006.01.023

[49] N. Matasovic, "Seismic Stability of Flysch Slopes", Gradjeviner, vol. 41, n. ${ }^{\circ}$, 1989.

[50] Presidencia de la República de Colombia, Decreto 926 del 19 de marzo de 2010, Requisitos de carácter técnico y científico para construcciones sismorresistentes NSR-10. Diario Oficial n. ${ }^{\circ}$ 47663. Disponible en https:// www.funcionpublica.gov.co/eva/gestornormativo/ norma.php? $\mathrm{i}=39255$

[51] R. C. Wilson y D. K. Keefer, "Dynamic Analysis of a Slope Failure from the 6 August 1979 Coyote Lake, California, Earthquake”, Int. J. Rock Mech. Min. Sci. Geomech. Abstr., vol. 21, n. ${ }^{\circ}$ 6, pp. 220-221, 1984. DoI: https://doi.org/10.1016/0148-9062(84)90499-6

[52] R. W. Jibson, "Regression Models for Estimating Coseismic Landslide Displacement”, Eng. Geol., vol. 91, n. ${ }^{\circ} 2-4$, pp. 209-218, 2007. DoI: https://doi.org/10.1016/j. enggeo.2007.01.013

[53]R. W. Jibson y J. A. Michael, Maps Showing Seismic Landslide Hazards in Anchorage, Alaska, Reston, Virginia: u.s. Geological Survey, 2009. Disponible en https://pubs.usgs.gov/sim/3077/downloads/3077_ pamphlet_508.pdf

[54] T. L. Tsai y H. F. Chen, "Effects of Degree of Saturation on Shallow Landslides Triggered by Rainfall", Environ. Earth Sci., vol. 59, n. ${ }^{\circ}$ 6, pp. 1285-1295, 2010. DoI: https://doi.org/10.1007/s12665-009-0116-3

[55]L. Montrasio, R. Valentino y C. Meisina, "Soil Saturation and Stability Analysis of a Test Site Slope Using the Shallow Landslide Instability Prediction (slip) Model”, Geotech. Geol. Eng., vol. 36, n. ${ }^{\circ}$ 4, pp. 2331-2342, 2018. DoI: https://doi.org/10.1007/s10706-018-0465-3 